\title{
Meeting the Demands of the Affordable Care Act: Improving Access to Primary Care
}

\author{
Peter M. McGough, MD, ${ }^{1,2}$ Thomas E. Norris, MD, \\ John D. Scott, MD, MSc, FIDSA, ${ }^{1,2}$ and Tim G. Burner, MD ${ }^{2,4}$
}

\section{Introduction}

$\mathbf{T}$ $\checkmark$ he Affordable Care Act has expanded health coverage to upward of 16.4 million uninsured people. ${ }^{1} \mathrm{Al}-$ though this has brought needed health care services to millions, it also brought new access challenges to the US health care system. In addition, emerging accountable care health programs create expectations for more convenient access to care. These new contracts often specify same-day access for primary care to reduce preventable visits to emergency departments (EDs). Although the goals of providing the right care to the right patient at the right place and time are important goals, they pose new challenges for primary care. These problems have been compounded by the limited number of new providers choosing careers in primary care.

Given these challenges and constraints, what strategies exist for improving patient access to primary care while supporting the goals of continuity and coordination of care? This article outlines strategies being implemented by University of Washington (UW) Medicine and the UW Neighborhood Clinics (UWNC).

\section{Primary Care Access: What We Have Tried}

The traditional approach to expanding primary care access in a group has been to hire more primary care physicians ([PCPs], including nurse practitioners and physician assistants). Although this approach helped, over time the practice schedules became jammed again. Patients with acute problems that could not be worked in were told to go to another practice (or worse, the ED) for care, resulting in unhappy patients and higher medical bills.

Another strategy has been to expand clinic hours to include evening and weekend hours. Although popular with patients, this did not increase provider capacity and often resulted in provider dissatisfaction. Another approach entailed reserving a number of schedule slots that are not filled until the day of use. These same-day appointments accommodated urgent problems, but reduced the number of appointments available for routine visits or some of the same-day holds went unused. We also have tried assigning a provider of the day to handle all acute or urgent appointments.

An innovative model used by many groups has been to implement open or advanced access scheduling. This approach encourages patients to schedule appointments with their PCP on the same day or over the next week or 2, as opposed to months ahead. This approach requires close monitoring of patient panels for each PCP, and scheduling that emphasizes continuity with that PCP to be successful. Medical groups who have used this approach and have worked down their practice backlog have reported significant improvement in access.

All of these approaches have their pros and cons, but as more and more patients seek access to primary care, the strategies begin to falter, and there are a growing number of patients who also expect that care can be provided without an office visit.

\section{Patient-Centered Medical Homes and Advanced Access}

The patient-centered medical home $(\mathrm{PCMH})^{2}$ is helping focus attention on important domains of primary care (eg, population health, care management of chronic conditions) and has led to many innovations in care delivery.

One of the key components of a PCMH, as defined by the National Committee for Quality Assurance, is enhanced access. ${ }^{3}$ Enhanced access involves patient access to the following:

1. Their personal electronic health record through a secure Web portal for information about their care and health status.

2. Their care team for timely help with questions and advice by phone or secure e-messaging.

3. Routine or urgent care from providers with evening and weekend hours.

\footnotetext{
${ }^{1}$ University of Washington Neighborhood Clinics, University of Washington Medicine, Seattle, Washington.

${ }^{2}$ Department of Family Medicine, University of Washington Medicine, Seattle, Washington.

${ }^{3}$ Retired.

${ }^{4}$ University of Washington Neighborhood Clinic-Woodinville, Seattle, Washington.
}

(c) McGough et al, 2017; Published by Mary Ann Liebert, Inc. This Open Access article is distributed under the terms of the Creative Commons Attribution Noncommercial License (http://creativecommons.org/licenses/by-nc/4.0/) which permits any noncommercial use, distribution, and reproduction in any medium, provided the original author(s) and the source are credited. 
Many patients who call their provider do not want or need an appointment; they just have a question or want simple medical advice. Under fee for service, this was an uncompensated service and patients usually were advised to make an office appointment. Under new approaches to health care delivery and reimbursement, it is both care and costeffective to interact with the care team by phone or e-mail for many issues. As already noted, patients are accessing more of their electronic health records. Programs such as Epic's OpenNotes give patients access to all of their record-not just problem lists or immunization records but also the complete documentation of their visits by their provider. ${ }^{4}$ Through this improved access to chart information, many patients find that scheduling an appointment to ask a question is no longer necessary.

Timely access to advice from the care team is another strategy to improve access to information and reduce the need for a visit. Patients can discuss medical issues with clinical staff, often registered nurses, who help decide whether their concerns can be managed at home or, if they need to be seen, by triaging patients to the appropriate site or level of care.

In addition to developing access to information as mentioned, UWNC has built new clinics based on a team care approach to clinic design, ${ }^{5}$ which facilitates team-based care that supports enhanced access. This team approach adjusts the roles of all team members to a level at the top of their training and scope, while sharing the work and reducing the burden on the provider. This in turn enables the PCP to provide care more efficiently and in different ways (eg, by phone or secure e-mail).

Finally, our affiliation with UW Medicine has enabled UWNC to establish primary care residency training programs. This helps us train our future workforce using the team-based care approach and strategies that we have found effective. It also prepares our residency graduates for the future of primary care medical practice.

\section{Walk-In Urgent Care Embedded in Primary Care}

Walk-in urgent care provides another strategy to expand access to care for primary scope issues. Although some urgent care clinics are freestanding, UWNC has chosen to create urgent care clinics that are embedded in primary care clinics. Embedding urgent care allows sharing of primary care clinic space, which is underutilized in the evenings and on weekends, and laboratory and X-ray resources.

With 7-day access, patients are able to be seen for primary scope acuities at a lower cost than for an ED visit. Established patients in the practice have immediate access to their electronic medical record, and urgent care providers are able to interact electronically with the PCP to consult about patients.

One challenge we found has been patients (often younger) who liked the convenience and sought to get much of their care through urgent care. We try to educate these patients on the value of having a PCP and, when needed, schedule appointments for them to establish a relationship with a PCP. There are some important imperatives for including urgent care as part of an integrated health system. One is to reduce preventable ED visits for relatively minor health problems, especially for systems that are assuming risk for the cost of care. Another is to avoid losing patients to other competing providers or health systems to maintain market share and reduce fragmentation of care. Embedding urgent care into primary care improves the patient experience by allowing same-day primary care access, 7 days a week, within our health system. It also improves the quality of care by maintaining continuity and improved communication between primary and urgent care providers.

\section{Retail Clinics}

A more recent approach to expanding access is through the use of retail clinics, which are small walk-in clinics typically located in pharmacies, grocery stores, or other retail establishments. These clinics typically provide a limited scope of services that include simple acute problems and usually some immunizations, all for a low-cost fixed fee. The market growth of retail clinics is linked to patient demand for convenience and lower cost. Another driver of retail clinic growth is the goal of many large pharmacy chains to expand their role in the health care market, while assuring that they capture the opportunity to fill any prescriptions that are written.

Health systems also are exploring retail clinics to expand access and to accommodate patient expectations for convenient lower-cost care. This is often done through an alliance with a well-established pharmacy chain that provides the clinic space, while the health system manages the clinical services. This approach links the retail clinic to broader health services, ensuring follow-up and backup, usually through the use of a shared electronic health record. Another consideration for health systems is that building new clinics is expensive. Retail clinics are a strategy that integrated health systems can use to expand market share at a lower cost to the system.

\section{Virtual Care}

Finally, an emerging access strategy is the use of virtual care, which provides 24-hour/7-day access to PCPs by phone or Internet video for a limited range of minor urgent care issues such as rashes, cough, and conjunctivitis. In 2015, UW Medicine launched a program called the Virtual Clinic, a teleurgent care service, in partnership with a local telehealth company (Carena, Seattle, WA). Patients who access the Virtual Clinic speak with a PCP within 10-30 minutes. Approximately $70 \%$ of all Virtual Clinic visits are managed definitively online, and patients are charged $\$ 40$ on their credit card. The remaining $30 \%$ are referred to primary care, urgent care or, rarely, the ED. The Virtual Clinic uses virtual practice guidelines, based on best-practice protocols, to ensure the safest delivery of health care. Patients report they are very satisfied with this prompt, professional, and convenient service.

The Virtual Clinic experience has taught us valuable lessons. First, it is important to educate patients about the scope of virtual care services and what they should expect in terms of medication prescriptions. Virtual care providers do not prescribe controlled substances and are very conservative in prescribing antibiotics. If testing is needed to decide about antibiotic usage, patients may be directed to their PCP or urgent care. Next, it is important that the video technology be as user friendly as possible; new handheld devices are incorporating many of these video tools. Finally, developing virtual care will mean learning a lot about the value of search 
engine marketing for a young population highly connected to the Web.

Virtual visits hold great potential for primary care as telehealth does for the health system in general.

\section{Conclusion}

Expectations for primary care are growing, as are the challenges we face in meeting them. In seeking to provide better access, quality, and improved patient experience at a lower cost, UW Medicine and the UWNC are piloting a range of strategies. If implemented in isolation, each of these strategies likely might be unsuccessful in meeting our goals. The challenge is to implement all of the strategies in a wellintegrated manner that reinforces all of the goals. Although such an initiative will not be easy, it holds great promise for health systems and the patients we serve. It is critically important that these new approaches be subjected to ongoing research and process improvement to confirm their value and build on their potential for providing the right care to the right patient at the right place and time.

\section{Author Disclosure Statement}

Drs. McGough, Norris, Scott, and Burner declared no conflicts of interest with respect to the research, authorship, and/or publication of this article. The authors received no financial support for this article.

\section{References}

1. Blumenthal D, Abrams M, Nuzum R. The affordable care act at 5 years. N Engl J Med 2015;372:2451-2458.

2. American Academy of Family Physicians, American Academy of Pediatrics, American College of Physicians, \& American Osteopathic Association. Joint Principles of the Patient-Centered Medical Home. 2007. www.aafp.org/dam/ AAFP/documents/practice_management/pcmh/initiatives/ PCMHJoint.pdf. Accessed September 29, 2015.

3. National Committee for Quality Assurance. The Future of Patient Centered Medical Homes: The Foundation for a Better Health Care System. 2014.www.ncqa.org/Portals/0/ Public\%20Policy/2014\%20Comment\%20Letters/The_Future_ of_PCMH.pdf. Accessed December 14, 2015.

4. Delbanco T, Walker J, Bell SK, et al. Inviting patients to read their doctors notes: a quasi-experimental study and a look ahead. Ann Intern Med 2012;157:461-470.

5. McGough PM, Jaffy MB, Norris TE, Sheffield P, Shumway M. Redesigning your workspace to support team-based care. Fam Pract Manag 2013;20:20-24.

Address correspondence to:

Peter M. McGough, MD

University of Washington Neighborhood Clinics University of Washington Medicine

$1100 \mathrm{NE}$ 45th St, Suite 500

Campus Box 354943

Seattle, WA 98195

E-mail: pmcgough@uwpn.org 